\title{
Association between Flash Glucose Monitors and A1C: A Retrospective Pre-Post Analysis
}

Mustafa Tekarli, PharmD Candidate (Class of 2021) ${ }^{1}$; Kyle Turner, PharmD, BCACP2 ${ }^{2}$ Daniel Witt, PharmD, FCCP, BCPS ${ }^{2}$

${ }^{1}$ University of Utah College of Pharmacy; ${ }^{2}$ Department of Pharmacotherapy, University of Utah College of Pharmacy

\begin{abstract}
Background: Randomized controlled trials have investigated the effect of continuous glucose monitors on hemoglobin A1C; however, more evidence is needed to justify their use and expand insurance coverage. Additionally, there are few published studies investigating the A1C lowering effect of flash glucose monitors (FGMs) in broad diabetes populations with varying insulin requirements. This analysis aimed to help fill this gap in medical literature and help clinicians evaluate costs/benefits when considering FGMs for their patients with diabetes. Objectives: To determine the association between FGM use and A1C reduction in patients with type 1 or type 2 diabetes mellitus regardless of insulin dependence. Methods: Pharmacy dispensing records were used to identify patients for inclusion. Patients who received a FGM from a University of Utah pharmacy between July 7, 2018 and July 7, 2020 were included. Patients who did not receive at least an 84-day supply of FGM sensors or did not have a baseline or follow-up A1C were excluded. Baseline and follow-up A1Cs, defined as A1Cs that are within one year before and 3-12 months after the FGM dispense date, were collected for each patient. New diabetes medications within a six-month window of the initial FGM dispense date were also recorded. Outcome variables were collected before and after patients received their first FGM (pre-FGM vs. post-FGM, respectively). The primary outcome was the difference between baseline and follow-up A1C for each patient. Secondary outcomes were the difference in baseline and follow-up A1C for various clinical subgroups within the overall sample. Descriptive statistics were used to summarize baseline characteristics and outcome data. Paired Student's t-tests were used to evaluate outcome differences ( $\alpha=0.05)$. Results: Fifty-seven patients (50.8\% male; mean age: 49 years) were included. For the primary outcome, the average baseline and follow-up A1Cs were 9.33\% and 8.32\%, respectively for a difference of $-1.01 \%$ ([95\% Cl -1.31:-0.72]; $p<0.0001)$. Conclusions: The use of FGMs is associated with decreases in A1C within a cohort of patients at one health system. Further effort to determine impact of FGM on clinical and economic outcomes is warranted.
\end{abstract}

Keywords: flash glucose monitor; continuous glucose monitor; diabetes; interstitial fluid glucose; A1C

\section{INTRODUCTION}

Self-monitoring blood glucose is a crucial component of diabetes management. Traditionally, patients have used the fingerstick method (also termed invasive blood glucose monitoring) as their primary means for measuring blood glucose levels. However, invasive blood glucose monitoring is not without disadvantages. The necessary supplies can be expensive and inconvenient to carry in public, finger sticks can be painful, and frequent blood glucose testing can contribute to diabetes distress and treatment fatigue. ${ }^{1-6}$ Because of these shortcomings, the use of flash glucose monitoring systems is becoming more prominent. These devices are a more advanced form of self-monitoring blood glucose and have been shown to increase treatment satisfaction compared to invasive monitoring. ${ }^{7,8}$ Flash glucose monitors are a type of continuous glucose monitor (a more commonly used term); for simplicity, the term "flash glucose monitor" will be used to distinguish flash glucose monitors from traditional continuous glucose monitors for the remainder of this paper.

Corresponding author: Kyle Turner, PharmD, BCACP

Assistant Professor (Clinical)

Department of Pharmacotherapy

University of Utah College of Pharmacy

Email: kyle.turner@pharm.utah.edu
Continuous glucose monitoring systems utilize an implantable sensor that reads and transmits interstitial glucose levels to a patient's reader or smartphone every 5-15 minutes; this is contrary to invasive monitoring which only captures glucose levels in a single time point. Because the sensor is implantable, continuous glucose monitors have allowed patients to quickly and conveniently check their blood glucose levels without the need for finger sticks. Additionally, the use of continuous glucose monitors is associated with a decreased risk of hypoglycemia and improvements in A1C compared to standard care. ${ }^{9-11}$ However, continuous glucose monitor use is primarily limited by high costs and lack of insurance coverage. These limitations paved the way for flash glucose monitors, the next attempt at fine-tuning the way patients measure their blood glucose.

Similar to continuous glucose monitors, flash glucose monitors utilize implantable sensors to continuously measure blood glucose. The key difference is flash glucose monitors do not continuously send the information to the user. Rather, the information is only transmitted to the user when it is requested (i.e., "flashing" the reader or smartphone over the sensor). This method also allows patients to quickly and conveniently measure their blood glucose without the need for finger sticks and at a lower cost than continuous glucose monitors. Unfortunately, the literature evaluating flash glucose monitors is limited compared to continuous glucose monitors. Current studies show that flash glucose monitors positively impact the 
risk of hypoglycemia, treatment satisfaction, and quality of life in various diabetes populations. ${ }^{7,8,12-15}$ However, the impact of flash glucose monitors on $\mathrm{A} 1 \mathrm{C}$ reduction is not as wellestablished, particularly in broader diabetes populations with varying insulin requirements. This ambiguity in treatment effect makes it difficult for clinicians to evaluate the costs and benefits when considering flash glucose monitors for their patients. Therefore, the objective of this study is to determine the association of flash glucose monitor use and $\mathrm{A} 1 \mathrm{C}$ reduction in patients with type 1 or type 2 diabetes, regardless of insulin use.

\section{METHODS}

\section{Study Design}

A retrospective, single-center, pre-post analysis was performed in patients with diabetes who used University of Utah Health community pharmacies. Pharmacy dispensing records were used to identify patients for inclusion. Patients 18 to 85 years of age with type 1 or type 2 diabetes were included if they received a flash glucose monitor between July 7, 2018 and July 7,2020 . Patients were excluded if they did not receive at least 84 consecutive days' worth of flash glucose sensors as this indicated inconsistent use of the flash glucose sensors. A length of 84 days was chosen because three months of use would be required to most accurately reflect changes in $\mathrm{A} 1 \mathrm{C}$ and pharmacy systems adjudicate flash glucose sensor fills by factors of 14 (each sensor can be used for a maximum of 14 days). Patients were also excluded if they did not have at least one baseline $\mathrm{A} 1 \mathrm{C}$ or one follow-up $\mathrm{A} 1 \mathrm{C}$. Baseline and follow-up $A 1 C$ were defined as A1Cs that were within one year before and 3-12 months after the initial flash glucose monitor dispense date, respectively.

\section{Data Collection}

At least one baseline and one follow up A1C was collected for each patient. The number of new diabetes medications within six months before and six months after the initial flash glucose sensor dispense date were also recorded. New diabetes medications were stratified in three-month increments based on their timing relative to the initial flash glucose monitor dispense. For example, a new diabetes medication that was started four months prior to a patient's initial flash glucose monitor dispense was grouped into the "3-6 months prior" strata. Hypoglycemic events were also collected for each patient for the total period of time in which a flash glucose sensor was being used. Hypoglycemic events were adjudicated into two categories: 1) "severe hypoglycemia" which was defined as a hypoglycemic event requiring hospitalization, an emergency department visit, or an urgent care visit or 2) "mildmoderate hypoglycemia" which was defined as hypoglycemic events not requiring medical intervention but were reported to healthcare teams. Hypoglycemia events were further stratified into quarters based on when they occurred during the given time period. For example, if a patient used a flash glucose sensor for 12 months and had a hypoglycemic event in month 5 , then this event was stratified into "Quarter 2".
The number of instances during flash glucose sensor use in which diabetes medications were discontinued, dose increased, or dose decreased were recorded for each patient. The number of instances in which patients met with certified diabetes educators or were provided lifestyle counseling were also collected. Lifestyle counseling was defined as any education relating to diet and exercise given to a patient by a provider, pharmacist, or certified diabetes educator. All lab values and patient characteristics were identified using electronic health records. The study protocol was deemed exempt under exemption category 4(iii) by the University of Utah Institutional Review Board as patients were not contacted and will not be reidentified.

\section{Study Outcomes}

The primary outcome was the difference in baseline and followup A1Cs (herein referred to as pre- and post-FGM A1C, respectively). Secondary outcomes were the differences in preand post-FGM A1Cs with the analysis limited to each of the following characteristics: patients without new diabetes medications, patients with an average pre-FGM A1C of $\geq 8 \%$, patients with an average pre-FGM A1C of $\geq 10 \%$, patients with type 2 diabetes not using prandial insulin, patients using basalbolus insulin or pumps, and patients that were being followed by the University of Utah clinical pharmacy service during flash glucose monitor use.

\section{Statistical Analysis}

A sample size of 51 patients was needed to detect a difference of $1 \%$ between pre- and post-FGM A1C in terms of the primary outcome with $80 \%$ power and an alpha of 0.05 . Descriptive statistics such as proportions for categorical variables and mean with standard deviation for continuous variables were used to summarize baseline characteristics. Primary and secondary outcomes were compared using paired student's t-tests. Linear regression was performed to evaluate the association between time to follow-up A1C (days) and observed change in A1C. This association was further analyzed by comparing the average change in A1C of patients with follow-up A1Cs after 90-179 days and follow-up A1Cs after $\geq 180$ days using student's t-tests. All statistical analyses were performed using Microsoft Excel's Data Analysis software.

\section{Post-Hoc Analyses}

The rate of hypoglycemia events every six months was compared in patients that had A1C changes of $\leq-0.7 \%$ (more beneficial) to patients that had A1C changes of $>-0.7 \%$ (less beneficial). A cutoff of $-0.7 \%$ was used as this was deemed to be a clinically meaningful reduction in A1C. The same analysis was conducted for rate of medications added, rate of medications stopped, rate of medication dose increases, rate of medication dose decreases, rate of lifestyle counseling instances, and rate of visits with a certified diabetes educator. All analyses were standardized to rates of every six months to account for variations in total days' supply of sensors dispensed between patients. 


\section{RESULTS}

\section{Patient Characteristics}

A total of 688 flash glucose sensor dispenses were identified from 15 pharmacies for a total of 169 unique patients. Of these 169 patients, 70 were excluded for lack of baseline or follow-up A1C and 42 were excluded for not receiving at least 84 consecutive days' supply of flash glucose sensors resulting in a total of 57 included patients (Figure 1). The proportion of included patients that were male was $50.9 \%$ with an average age of 48.9 years $(S D=12.4)$ at the time of inclusion. Patients received an average of 330 days' worth of flash glucose sensors and 11 (19.3\%) had type 1 diabetes. There were few new diabetes medication starts within six months of the initial flash glucose monitor dispense (Table 1). Excluded patients had similar demographic and clinical baseline characteristics except for "patient pay amount per month". Patients that were included paid an average of \$28.97 per month and patients that were excluded paid an average of $\$ 53.92$ per month. All patients received the flash glucose monitoring system branded as FreeStyle Libre (no patients received FreeStyle Libre 2).

\section{Primary Analyses}

For the primary outcome, average pre-FGM A1C was $9.33 \%$ and average post-FGM A1C was $8.32 \%$ for a difference of $-1.01 \%$ $([95 \% \mathrm{Cl}-1.31:-0.72] ; \quad p<0.0001)$. Fifty patients $(87.7 \%)$ experienced a reduction in $\mathrm{A} 1 \mathrm{C}$ after initial flash glucose monitor dispense, and 23 (40.4\%) experienced an A1C reduction of at least $1 \%$ (Figure 2 ). The $A 1 C$ difference observed in the primary analysis was sustained across all of the following secondary analysis groups (Table 2 ): patients with no new diabetes medications ( $n=35$; difference of $-0.80 \%,[95 \% \mathrm{Cl}$ 1.12:-0.49]; $p<0.0001$ ), patients with baseline A1Cs of at least $8 \%(n=41$; difference of $-1.31 \%$, [95\%Cl $-1.65:-0.96] ; p<0.0001)$, patients with baseline A1Cs of at least $10 \%(n=17$; difference of $-1.75 \%,[95 \% \mathrm{Cl}-2.45:-1.05]$; $\mathrm{p}<0.0001$ ), patients with type 2 diabetes not using prandial insulin $(n=15$; difference of $-1.17 \%$, [95\% $\mathrm{Cl}-2.04:-0.30] ; p=0.01)$, patients who were using basalbolus insulin or insulin pumps $(n=42$; difference of $-0.95 \%$, [95\% $\mathrm{Cl}-1.23:-0.67] ; p<0.0001)$, and patients who were being followed by the clinical pharmacy service $(n=12$; difference of $1.02 \%,[95 \% \mathrm{Cl}-1.51:-0.52] ; \mathrm{p}<0.0001)$. Linear regression analysis showed no clear association between time to followup $A 1 C$ and change in $A 1 C\left(R^{2}=0.001\right.$; Figure 3$)$. In addition, there was not a statistically significant difference in $\mathrm{A} 1 \mathrm{C}$ change in patients that had a follow-up A1C after 90-179 days ( $n=36$; mean change in $\mathrm{A} 1 \mathrm{C}$ of $-1.0 \%)$ and patients that had a followup $\mathrm{A} 1 \mathrm{C}$ after $\geq 180$ days ( $\mathrm{n}=21$; mean change in $\mathrm{A} 1 \mathrm{C}$ of $-1.1 \%$ | $p=0.61)$.

\section{Post-Hoc Analyses}

Of the 57 total patients, 2 (3.5\%) experienced severe hypoglycemia. Between these 2 patients, there were 5 total occurrences (four were experienced by 1 patient). Twenty-six patients (45.6\%) reported mild-moderate hypoglycemia; there were 47 total events between the 26 patients. Of the 47 mildmoderate hypoglycemic events, 24 (51.1\%) occurred within the first quarter of flash glucose sensor use, 11 (23.4\%) occurred within the second quarter, $11(23.4 \%)$ within the third quarter, and $7(14.9 \%)$ within the fourth quarter. Patients that experienced an A1C change of $\leq-0.7 \%(n=31)$ did not have a statistically significant difference in rate of mild-moderate hypoglycemic events (0.48 events $/ 6$ months) compared to patients that had an $\mathrm{A} 1 \mathrm{C}$ change of $>-0.7 \%$ ( $n=26,0.41$ events 6 months; $p=0.67)$. Patients with $A 1 C$ changes of $\leq-$ $0.7 \%$ had a higher rate of medication additions ( 0.16 vs 0.11 ), dose increases (1.14 vs 0.69 ), dose decreases (0.53 vs 0.28 ), lifestyle counseling ( 0.81 vs 0.51$)$, and meetings with certified diabetes educators ( 0.24 vs 0.03$)$, though none of these differences were statistically significant (Table 3). However, when all interventions were pooled into a single analysis, patients with larger $\mathrm{A} 1 \mathrm{C}$ reductions showed a statistically significant increase in rate of receiving any intervention compared to patients with lower A1C reductions (2.95 vs. 1.73; $p=0.05)$.

\section{DISCUSSION}

Among adult patients with type 1 or type 2 diabetes from a single healthcare system, the use of flash glucose monitors was associated with a decrease in A1C, regardless of prandial insulin use. This $\mathrm{A} 1 \mathrm{C}$ benefit was consistently observed across various clinical subgroups. These findings are significant as they add to the growing body of evidence that supports the beneficial effects of flash glucose monitors on glycemic control. ${ }^{16-19}$ Additionally, it is the first to utilize a pre-post design as a means to mitigate potential bias and confounding.

Notably, the observed $\mathrm{A} 1 \mathrm{C}$ decrease was sustained in patients that were not started on new diabetes medications within 6 months before or after flash glucose monitor initiation. This result suggests that reductions in A1C associated with flash glucose monitor initiation are independent of new diabetes medications. This sustained reduction in A1C, despite lack of new medications, may be due to patients checking their blood glucose more frequently because of a more convenient means for glucose monitoring. Increases in frequency of blood glucose self-monitoring may have aided patients in identifying foods that significantly increase their blood glucose resulting in better diet selections thereafter. For patients that are on insulin and have the autonomy to make independent dose adjustments, more frequent blood glucose self-monitoring may have led to more informed decision-making regarding dose adjustments resulting in improved glycemic control. These findings suggest that flash glucose monitors have the potential to independently decrease $\mathrm{A} 1 \mathrm{C}$ in various diabetes populations.

Most of the analyzed subgroups achieved a clinically relevant reduction in $\mathrm{A} 1 \mathrm{C}$ of at least $1 \%$. The only subgroups that did not achieve at least a $1 \%$ reduction in $\mathrm{A} 1 \mathrm{C}$ were patients that did not have any new diabetes medications and the group of patients on basal-bolus insulin; these groups achieved A1C reductions of $0.80 \%$ and $0.95 \%$, respectively. However, it is worth noting that the "no new diabetes medications" subgroup 
had the third lowest average baseline A1C (9.0\%); this likely contributed to the smaller effect size observed relative to the other subgroups. As for the basal-bolus insulin subgroup, the small magnitude in $\mathrm{A} 1 \mathrm{C}$ reduction relative to the other subgroups was surprising. It was originally expected that patients who were using multiple daily insulin injections would achieve the greatest benefit from flash glucose monitors compared to the other subgroups because this subgroup would be able to tailor their insulin doses based on their glucose readings. Nonetheless, this group still achieved a clinically relevant reduction in A1C. Overall, the magnitude of $\mathrm{A} 1 \mathrm{C}$ reduction was clinically meaningful in all the groups as the 2020 American Diabetes Association guidelines state that noninsulin agents added to initial therapy will generally lower $\mathrm{A} 1 \mathrm{C}$ by $0.7-$ $1.0 \% .^{20,21}$ Therefore, these findings suggest that the use of flash glucose monitors has the potential to replace initiation of another diabetes medication. This may result in decreased pillburden, injections, medication side effects, and healthcare costs (28-day supply of flash glucose monitor sensors $=\$ 115$ $\$ 130$; 28-day supply of GLP-1 agonists $=\$ 650-\$ 900 ; 28$-day supply of SGLT-2 inhibitors $=\$ 500-\$ 550) .{ }^{22-24}$ However, clinicians must also consider the cardiovascular and renal protective benefits that GLP-1 agonists and SGLT-2 inhibitors may provide as these benefits are unlikely to be seen with flash glucose monitor use. Additionally, simply starting a patient on a flash glucose monitoring system is likely not enough to result in a clinically meaningful reduction in A1C. In this study's population, patients and their healthcare teams used information from the flash glucose monitoring systems to facilitate addition or removal of medications, adjustments in medication doses, and changes in lifestyle. This study also suggests that patients using flash glucose monitoring systems who are receiving more interventions and follow up with their healthcare team will experience greater reductions in A1C compared to patients receiving fewer interventions and follow up. Ultimately, flash glucose monitoring systems should function to provide the patient and their healthcare team with more information to make better-informed decisions and prevent clinical inertia.

Another notable finding was within the subgroup of patients that was being followed by the University of Utah's clinical pharmacy service. Clinical pharmacists in this service practice under a collaborative practice agreement that allows them to initiate, discontinue, and change medications and doses. Patients followed by the clinical pharmacy service are those that are referred by University of Utah providers. All patients are seen by a clinical pharmacist for an initial visit in which information is collected, medications are reconciled, and an initial treatment plan is formulated (approximately 30-60 minutes in length). Thereafter, patients are contacted for follow-up at varying timeframes depending on the clinical scenario. Although the clinical pharmacy subgroup was small in this study $(n=12)$, patients in this subgroup achieved an average A1C reduction of $1.02 \%$, despite being tied for the lowest average baseline $\mathrm{A} 1 \mathrm{C}$ compared to all other groups. Once again, these findings suggest that frequent healthcare provider involvement, follow-up, and intervention (particularly by a pharmacist) may optimize $\mathrm{A} 1 \mathrm{C}$ reduction in patients using flash glucose monitors; the resulting effect on $\mathrm{A} 1 \mathrm{C}$ may be enough to justify the costs of a flash glucose monitor and sensors. However, further research assessing cost-effectiveness is required to support this claim.

Hypoglycemia is an important factor that must be considered when managing diabetes. The results of this analysis show that the rate of experiencing mild-to-moderate hypoglycemic events is not increased with larger A1C reductions in patients using flash glucose monitoring systems. This finding may be attributable to patients being able to check their blood glucose levels more readily and more frequently leading to measures being taken to prevent hypoglycemia. Additionally, it seems that the incidence of hypoglycemic events begins to decline as patients continue to use their flash glucose monitoring systems; this is supported by the decreasing number of hypoglycemic events as patients progressed from quarter 1 of flash glucose sensor use (24 events/47 total events; $51.1 \%$ ) to quarter 4 of flash glucose sensor use (7 events/47 total events; 14.9\%). These results are consistent with current literature suggesting that the use of flash glucose monitoring systems decrease the incidence of hypoglycemic events. ${ }^{12-15}$

This study is not without limitations. First, this was a singlecenter study and may not be generalizable to other settings. Second, investigators were unblinded during data collection and this may have introduced investigator bias and exaggerated treatment effect sizes. Third, this study is inherently subject to confounding due to the nature of the study design. Although steps were taken to control for as many confounding variables as possible (e.g. baseline characteristics, new medications), residual confounding likely remains. Fourth, the temporal relationship between the baseline and follow-up A1Cs in this study is not as strong relative to prospective studies. Investigators attempted to mitigate this by collecting multiple A1Cs and using averages when appropriate. Fifth, many of the subgroup analyses contained small samples; this resulted in wide confidence intervals and may have inflated effect sizes. Sixth, patients in this study that were receiving healthcare interventions more frequently experienced larger reductions in A1C. However, this result may have been exaggerated by healthy-user bias; that is, patients that are more willing to see their healthcare team and accept healthcare interventions may be more likely to take other measures to lower their A1C. Seventh, this analysis may have been subject to selection bias; because this study excluded patients who did not receive flash glucose sensors for at least 84 days, patients that could not afford to purchase sensors for extended periods of time may have been inadvertently excluded. This is further supported by the disparity in patient pay amounts between included and excluded patients. Lastly, the pre-post design used in this study inherently predisposes the post-FGM data to prolonged disease burden and progression of diabetes compared to the pre-FGM 
data simply because the post-FGM data was analyzed later in time. This may have resulted in an underestimation of the true treatment effect since diabetes is more difficult to control when it has progressed.

\section{CONCLUSION}

The use of flash glucose monitors is associated with decreases in $\mathrm{A} 1 \mathrm{C}$ within a cohort of patients at one healthcare system. Further effort to determine impact of flash glucose monitors on clinical and economic outcomes is warranted.

Disclosures: The authors have no conflicts of interest to disclose. This manuscript is not under consideration elsewhere and has not been previously published in a similar form.

Treatment of Human Subjects: IRB exemption granted

\section{REFERENCES}

1. Ong WM, Chua SS, Ng CJ. Barriers and facilitators to self-monitoring of blood glucose in people with type 2 diabetes using insulin: a qualitative study. Patient Prefer Adherence. 2014;8:237-246.

2. Hortensius J, Kars MC, Wierenga WS, Kleefstra N, Bilo $\mathrm{HJ}$, van der Bijl JJ. Perspectives of patients with type 1 or insulin-treated type 2 diabetes on self-monitoring of blood glucose: a qualitative study. BMC Public Health. 2012;12:167.

3. Ugwu ET, Orjioke CJG, Young EE. Self Monitoring of Blood Glucose Among Patients with Type 2 Diabetes Mellitus in Eastern Nigeria: Need for Multi-strategic Interventions. Curr Diabetes Rev. 2018;14(2):175-181.

4. Scorpiglione N, el-Shazly M, Abdel-Fattah M, et al. Epidemiology and determinants of blood glucose selfmonitoring in clinical practice. Diabetes Res Clin Pract. 1996;34(2):115-125.

5. Mastura I, Mimi O, Piterman L, Teng CL, Wijesinha S. Self-monitoring of blood glucose among diabetes patients attending government health clinics. Med J Malaysia. 2007;62(2):147-151.

6. Snoek F, Malanda U, de Wit M. Self-monitoring of blood glucose: psychological barriers and benefits. In: Taylor \& Francis; 2008.

7. Al Hayek AA, Al Dawish MA. The Potential Impact of the FreeStyle Libre Flash Glucose Monitoring System on Mental Well-Being and Treatment Satisfaction in Patients with Type 1 Diabetes: A Prospective Study. Diabetes Ther. 2019;10(4):1239-1248.

8. Yaron M, Roitman E, Aharon-Hananel G, et al. Effect of Flash Glucose Monitoring Technology on Glycemic Control and Treatment Satisfaction in Patients With Type 2 Diabetes. Diabetes Care. 2019;42(7):11781184.
9. Beck RW, Riddlesworth T, Ruedy K, et al. Effect of Continuous Glucose Monitoring on Glycemic Control in Adults With Type 1 Diabetes Using Insulin Injections: The DIAMOND Randomized Clinical Trial. Jama. 2017;317(4):371-378.

10. Lind M, Polonsky W, Hirsch IB, et al. Continuous Glucose Monitoring vs Conventional Therapy for Glycemic Control in Adults With Type 1 Diabetes Treated With Multiple Daily Insulin Injections: The GOLD Randomized Clinical Trial. Jama. 2017;317(4):379-387.

11. Janapala RN, Jayaraj JS, Fathima N, et al. Continuous Glucose Monitoring Versus Self-monitoring of Blood Glucose in Type 2 Diabetes Mellitus: A Systematic Review with Meta-analysis. Cureus. 2019;11(9):e5634.

12. Haak T, Hanaire H, Ajjan R, Hermanns N, Riveline JP, Rayman G. Use of Flash Glucose-Sensing Technology for 12 months as a Replacement for Blood Glucose Monitoring in Insulin-treated Type 2 Diabetes. Diabetes Ther. 2017;8(3):573-586.

13. Bolinder J, Antuna R, Geelhoed-Duijvestijn P, Kröger J, Weitgasser R. Novel glucose-sensing technology and hypoglycaemia in type 1 diabetes: a multicentre, nonmasked, randomised controlled trial. Lancet. 2016;388(10057):2254-2263.

14. Ogawa W, Hirota $Y$, Osonoi T, et al. Effect of the FreeStyle Libre(TM) Flash Glucose Monitoring System on Glycemic Control in Subjects with Type 2 Diabetes Treated with Basal-Bolus Insulin Therapy: An Open Label, Prospective, Multicenter Trial in Japan. J Diabetes Investig. 2020.

15. Pintus D, Ng SM. Freestyle libre flash glucose monitoring improves patient quality of life measures in children with Type 1 diabetes mellitus (T1DM) with appropriate provision of education and support by healthcare professionals. Diabetes Metab Syndr. 2019;13(5):2923-2926.

16. Wada E, Onoue T, Kobayashi T, et al. Flash glucose monitoring helps achieve better glycemic control than conventional self-monitoring of blood glucose in non-insulin-treated type 2 diabetes: a randomized controlled trial. BMJ Open Diabetes Res Care. 2020;8(1).

17. Furler J, O'Neal D, Speight J, et al. Use of professionalmode flash glucose monitoring, at 3-month intervals, in adults with type 2 diabetes in general practice (GPOSMOTIC): a pragmatic, open-label, 12-month, randomised controlled trial. Lancet Diabetes Endocrinol. 2020;8(1):17-26. 
18. Fokkert $M$, van Dijk $P$, Edens $M$, et al. Improved wellbeing and decreased disease burden after 1-year use of flash glucose monitoring (FLARE-NL4). BMJ Open Diabetes Res Care. 2019;7(1):e000809.

19. Paris I, Henry C, Pirard F, Gérard AC, Colin IM. The new FreeStyle libre flash glucose monitoring system improves the glycaemic control in a cohort of people with type 1 diabetes followed in real-life conditions over a period of one year. Endocrinol Diabetes Metab. 2018;1(3):e00023.

20. Pharmacologic Approaches to Glycemic Treatment: Standards of Medical Care in Diabetes-2019. Diabetes Care. 2019;42(Suppl 1):S90-s102.

21. Bennett WL, Maruthur NM, Singh S, et al. Comparative effectiveness and safety of medications for type 2 diabetes: an update including new drugs and 2-drug combinations. Ann Intern Med. 2011;154(9):602-613.

22. G. Freestyle Libre. https://www.goodrx.com/freestyle-libre. Published 2020. Accessed 8-8-2020, 2020.

23. G. Victoza. https://www.goodrx.com/victoza. Published 2020. Accessed 8-8-2020, 2020.

24. G. Jardiance. https://www.goodrx.com/jardiance. Published 2020. Accessed 8-8-2020, 2020. 
Figure 1. Eligibility and Group Assignment

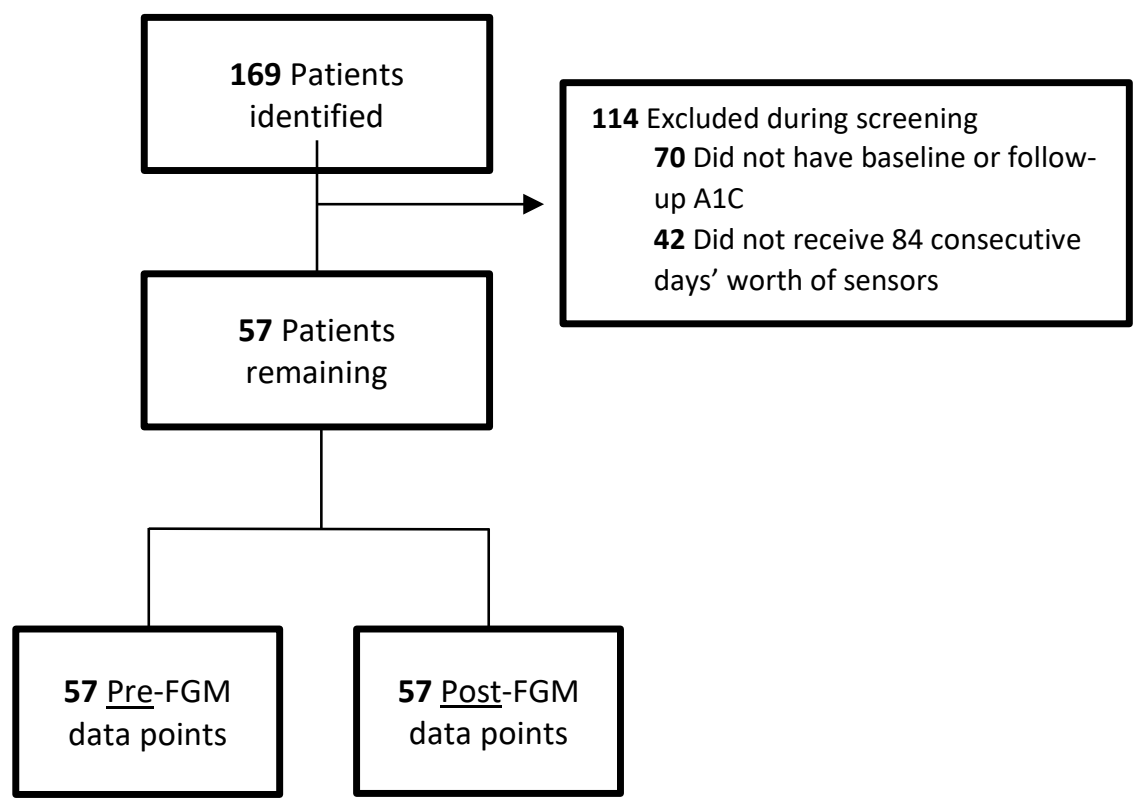

Figure 2 - Change in A1C for all Included Patients

\section{Primary Outcome}

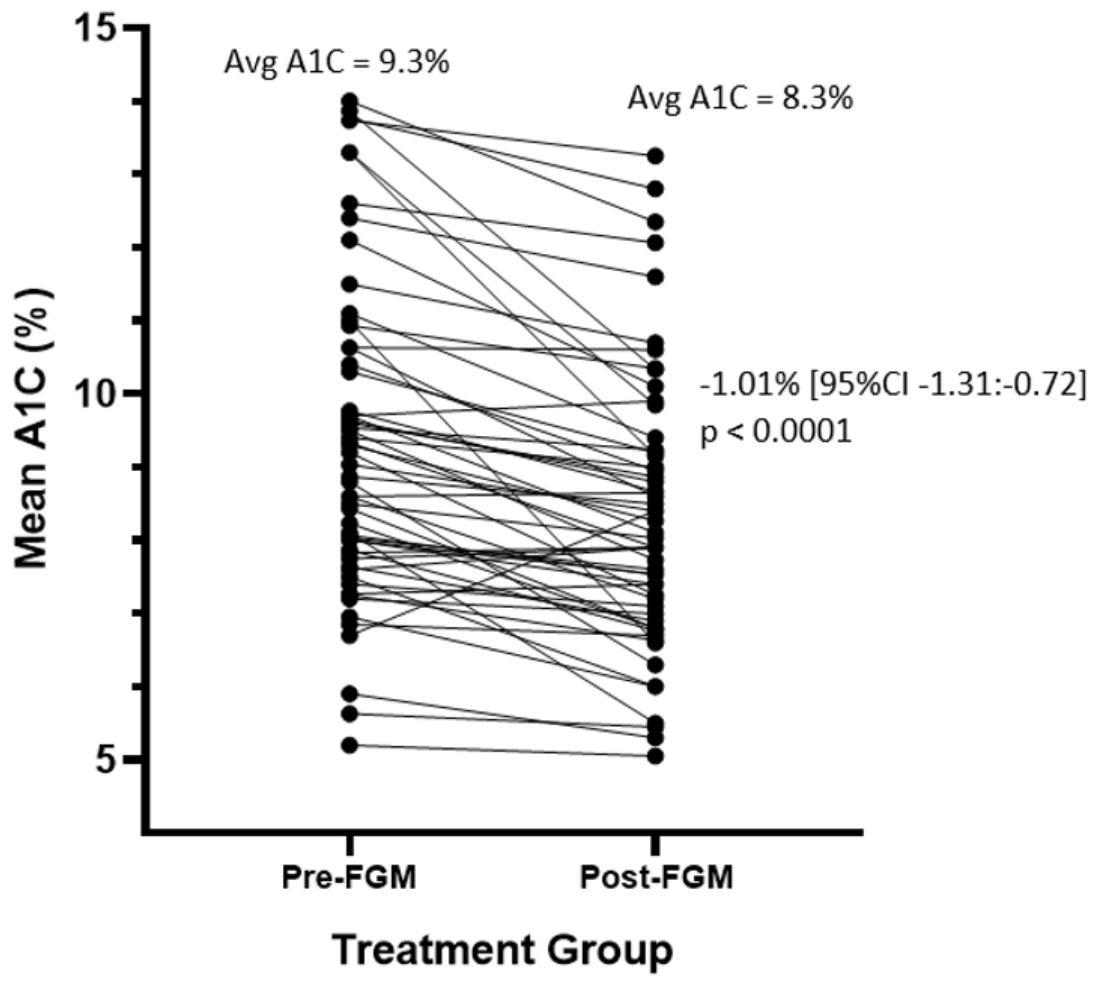


Figure 3. Regression Analysis of Change in A1C and Time to Follow-up A1C

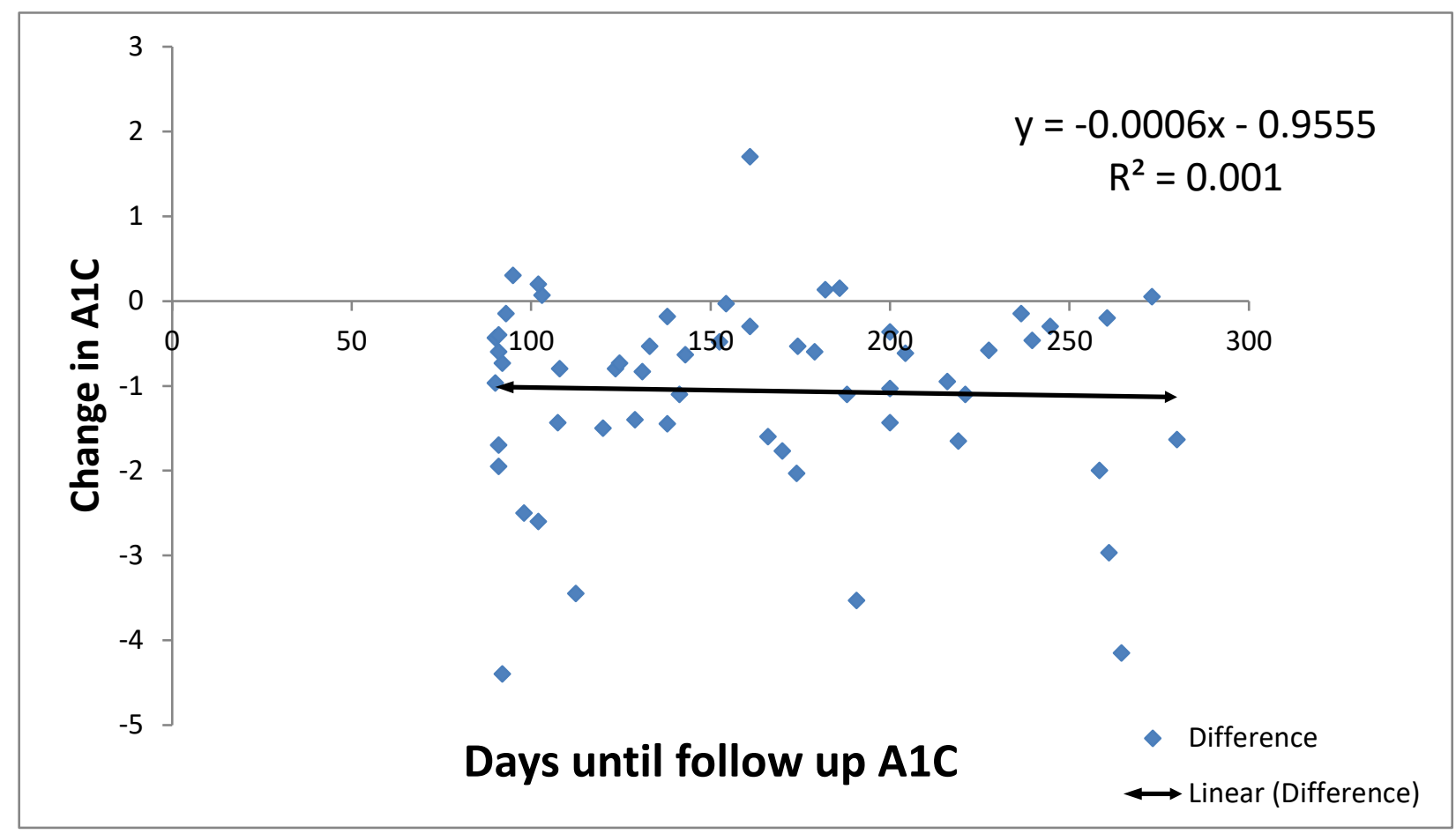


Table 1. Patient Demographics and Clinical Variables

\begin{tabular}{|c|c|c|}
\hline Variable & $\begin{array}{l}\text { Included Patients } \\
\quad(\mathbf{N}=\mathbf{5 7})\end{array}$ & $\begin{array}{l}\text { Excluded Patients } \\
\qquad(\mathrm{N}=112)\end{array}$ \\
\hline Mean age (SD), years & 49.9 (12.4) & $50.0(14.4)$ \\
\hline Male, no. (\%) & $29(50.9)$ & $61(54.5)$ \\
\hline \multicolumn{3}{|l|}{ Ethnicity, no. (\%) } \\
\hline Hispanic/Latino & $7(12.3)$ & $17(15.2)$ \\
\hline Caucasian & $45(79.0)$ & $79(70.5)$ \\
\hline African American & $0(0.0)$ & $4(3.6)$ \\
\hline Asian & $1(1.8)$ & $3(2.7)$ \\
\hline Other & $4(7.0)$ & $9(8.0)$ \\
\hline Type 1 diabetes, no. (\%) & $11(19.3)$ & $25(22.3)$ \\
\hline \multicolumn{3}{|l|}{ On basal + bolus insulin or pump, no. (\%) } \\
\hline Basal-bolus & $39(68.4)$ & $59(52.7)$ \\
\hline Insulin Pump & $3(5.3)$ & $5(4.5)$ \\
\hline On basal insulin without bolus, no. (\%) & $10(17.5)$ & $19(17.0)$ \\
\hline Mean sensor days dispensed, no. (SD) & $329.9(165.3)$ & $103.3(106.3)$ \\
\hline \multicolumn{2}{|l|}{$\begin{array}{l}\text { Number of patients with } \geq 1 \text { DM medications } \\
\text { within } 6 \text { months of sensor dispense (\%) }\end{array}$} & N/A \\
\hline 3-6 months prior & $8(14.0)$ & \\
\hline $1-3$ months prior & $6(10.5)$ & \\
\hline 1 month prior & $6(10.5)$ & \\
\hline 1 month after & $3(5.3)$ & \\
\hline 1-3 months after & $2(3.5)$ & \\
\hline 3-6 months after & $3(5.3)$ & \\
\hline \multicolumn{2}{|l|}{$\begin{array}{l}\text { Number of patients with } \geq 2 \text { DM medications } \\
\text { within } 6 \text { months of sensor dispense (\%) }\end{array}$} & N/A \\
\hline 3-6 months prior & $2(3.5)$ & \\
\hline 1-3 months prior & $0(0)$ & \\
\hline 1 month prior & $0(0)$ & \\
\hline 1 month after & $0(0)$ & \\
\hline 1-3 months after & $1(1.8)$ & \\
\hline 3-6 months after & $0(0)$ & \\
\hline Mean number of baseline A1Cs & 2.56 & $\mathrm{~N} / \mathrm{A}$ \\
\hline Mean number of follow-up A1Cs & 1.91 & $\mathrm{~N} / \mathrm{A}$ \\
\hline \multicolumn{3}{|l|}{ Non-insulin medications, no. (\%) } \\
\hline Metformin & $33(57.9)$ & $64(57.1)$ \\
\hline Sulfonylurea & $4(7.0)$ & $19(17.0)$ \\
\hline TZD & $3(5.3)$ & $7(6.3)$ \\
\hline GLP-1 agonist & $12(21.1)$ & $32(28.6)$ \\
\hline SGLT-2 Inhibitors & $12(21.1)$ & $28(25.0)$ \\
\hline DPP-IV Inhibitors & $3(5.3)$ & $13(11.6)$ \\
\hline Followed by Clinical Pharmacy Service, no. (\%) & $12(21.1)$ & $29(25.9)$ \\
\hline Average patient pay amount per month, USD & 28.97 & 53.92 \\
\hline Average days until follow-up A1C, days (SD) & 162.0 & $\mathrm{~N} / \mathrm{A}$ \\
\hline Used CGM prior to FGM, no. (\%) & $1(1.8)$ & $2(1.8)$ \\
\hline
\end{tabular}

$\mathrm{SD}=$ standard deviation, $\mathrm{DM}=$ diabetes mellitus, $\mathrm{A} 1 \mathrm{C}=$ glycosylated hemoglobin, $\mathrm{TZD}=$ thiazolidinediones, GLP-1 = glucagon-like peptide-1, SGLT-2 = sodium-glucose cotransporter-2, DPP-IV = dipeptidyl peptidase-4, USD = U.S. Dollar, $\mathrm{CGM}=$ continuous glucose monitor, $\mathrm{FGM}=$ flash glucose monitor, $\mathrm{N} / \mathrm{A}=$ not applicable 
Table 2. Primary and Secondary Outcomes

\begin{tabular}{|c|c|c|c|c|}
\hline Outcome & $\begin{array}{l}\text { Pre- } \\
\text { FGM }\end{array}$ & $\begin{array}{l}\text { Post- } \\
\text { FGM }\end{array}$ & Difference $[95 \% \mathrm{CI}]$ & p-value \\
\hline $\begin{array}{l}\text { Primary Outcome } \\
\text { Average A1C (all patients, } \\
\mathrm{n}=57 \text { ) }\end{array}$ & $9.33 \%$ & $8.32 \%$ & $\begin{array}{l}-1.01 \%[95 \% \mathrm{CI}- \\
1.31:-0.72]\end{array}$ & $<0.0001$ \\
\hline $\begin{array}{l}\text { Secondary Outcomes } \\
\text { Average A1C } \\
\text { No new DM meds ( } \mathrm{n}= \\
\text { 35) } \\
\text { Baseline A1C } \geq 8 \% \text { ( } \mathrm{n}= \\
41 \text { ) } \\
\text { Baseline A1C } \geq 10 \% \text { ( } \mathrm{n}= \\
17) \\
\text { T2DM pts not on prandial } \\
\text { insulin ( }=15) \\
\text { Basal-bolus or pump ( } \mathrm{n} \\
=42) \\
\text { Clinical pharmacy pts. (n } \\
=12)\end{array}$ & $\begin{array}{l}9.00 \% \\
10.23 \% \\
12.09 \% \\
8.81 \% \\
\\
9.52 \% \\
8.86 \%\end{array}$ & $\begin{array}{l}8.20 \% \\
8.92 \% \\
10.35 \% \\
7.64 \% \\
8.56 \% \\
7.85 \%\end{array}$ & $\begin{array}{l}-0.80 \%[95 \% \mathrm{CI}- \\
1.12:-0.49] \\
-1.31 \%[95 \% \mathrm{CI}- \\
1.65:-0.96] \\
-1.75 \%[95 \% \mathrm{CI}- \\
2.45:-1.05] \\
-1.17 \%[95 \% \mathrm{CI}- \\
2.04:-0.30] \\
\\
-0.95 \%[95 \% \mathrm{CI}- \\
1.23:-0.67] \\
-1.02 \%[95 \% \mathrm{CI}- \\
1.51:-0.52]\end{array}$ & $\begin{array}{r}<0.0001 \\
<0.0001 \\
<0.0001 \\
0.0100 \\
<0.0001 \\
<0.0001\end{array}$ \\
\hline
\end{tabular}

$\mathrm{FGM}=$ flash glucose monitor, $\mathrm{CI}=$ confidence interval, $\mathrm{A} 1 \mathrm{C}=$ glycosylated hemoglobin, $\mathrm{DM}=$ diabetes mellitus, meds $=$ medications, $\mathrm{pts}=$ patients, $\mathrm{T} 2 \mathrm{DM}=$ type 2 diabetes mellitus

Table 3. Results of Post-Hoc Analyses

\begin{tabular}{|c|c|c|c|}
\hline Outcome $^{¥}$ & $\begin{array}{l}\text { A1C } \Delta \leq- \\
0.7 \% \\
(n=31)\end{array}$ & $\begin{array}{l}\text { A1C } \Delta>- \\
0.7 \% \\
(n=26)\end{array}$ & $\begin{array}{l}\text { p- } \\
\text { value }\end{array}$ \\
\hline $\begin{array}{l}\text { Mild-moderate } \\
\text { hypoglycemia }\end{array}$ & 0.48 & 0.41 & 0.68 \\
\hline Medication added & 0.16 & 0.11 & 0.61 \\
\hline Medication stopped & 0.06 & 0.09 & 0.73 \\
\hline Dose increased & 1.14 & 0.69 & 0.25 \\
\hline Dose decreased & 0.53 & 0.28 & 0.23 \\
\hline $\begin{array}{l}\text { Received lifestyle } \\
\text { counseling }\end{array}$ & 0.81 & 0.51 & 0.15 \\
\hline Met with CDE & 0.24 & 0.03 & 0.08 \\
\hline Any Intervention & 2.95 & 1.73 & 0.05 \\
\hline
\end{tabular}

